\title{
HIGH PERFORMANCE ERASURE CODING FOR VERY LARGE STRIPE SIZES
}

\author{
Walker Haddock \\ Purushotham V. Bangalore \\ Department of Computer Science \\ University of Alabama at Birmingham \\ Birmingham, AL, USA \\ \{whaddock,puri\}@uab.edu
}

\author{
Matthew L. Curry \\ Center for Computing Research \\ Sandia National Laboratories \\ Albuquerque, NM, USA \\ mlcurry@sandia.gov
}

\author{
Anthony Skjellum \\ SimCenter \\ University of Tennessee at Chattanooga \\ Chattanooga, TN, USA \\ tony-skjellum@utc.edu
}

\begin{abstract}
Exascale computing demands high bandwidth and low latency I/O on the computing edge. Object storage systems can provide higher bandwidth and lower latencies than tape archive. File transfer nodes present a single point of mediation through which data moving between these storage systems must pass. By increasing the performance of erasure coding, stripes can be subdivided into large numbers of shards.

This paper's contribution is a prototype nearline disk object storage system based on Ceph. We show that using general purpose graphical processing units (GPGPU) for erasure coding on file transfer nodes is effective when using a large number of shards. We describe an architecture for nearline disk archive storage for use with high performance computing (HPC) and demonstrate the performance with benchmarking results. We compare the benchmark performance of our design with the Intel ${ }^{\circledR}$ Storage Acceleration Library (ISA-L) CPU based erasure coding libraries using the native Ceph erasure coding feature.
\end{abstract}

Keywords: GPGPU, erasure coding, storage, object, resilience, fault tolerance.

\section{INTRODUCTION}

The world's largest supercomputers are rapidly approaching exascale and their demands for I/O are increasing proportionately. The current performance of the Summit supercomputer is 200 PFs (McCorkle 2018) and the Aurora exascale system is planned to be online in 2021 (Morgan, T. P. 2018). Systems that achieve exascale will be 50 times faster than the $20 \mathrm{PF}$ supercomputers of today. The size of the memory in these supercomputers will increase proportionately, requiring the I/O bandwidth to keep pace. Burst Buffers (BB) have provided the technology to meet the I/O bandwidth and latency requirements for the top tier of storage and provide the interface to the larger but slower parallel file systems (PFS), like Lustre, as the second tier of the high performance storage. Curated data products from the jobs executed on these massive systems 
may be eventually stored on lower performance tape archives where increases in bandwidth are more difficult to achieve. Object storage systems have met the capacity, reliability and economical requirements of cloud service providers for over a decade and can be used to interface between the higher performance PFSs in HPC to the lower performance tape archive storage system. The use of object storage as a nearline disk archive (NLDA) storage system has been demonstrated by the Trinity supercomputer (Morton 2017). Exascale systems are built with more components than previous generations which results in a shorter time to failure (Bent, Settlemeyer, and Grider 2016). In order for applications to make forward progress on large supercomputer clusters, checkpoint/restart (CR) allows applications to restart in the event of a failure caused by a hardware malfunction. Having larger problem sizes made possible by the greater memory and greater compute resources leads to larger CR data sizes. Storing CR datasets for a sufficient period of time will be most economical on NLDA systems (Grider, G 2016) which may also reduce demand for CR storage on tape.

The high performance tier for exascale storage consists of BBs and PFSs which are expensive to acquire and operate. The traditional memory hierarchy pyramid shows that smaller amounts of the more expensive memory is put at the top of the pyramid with increasingly larger amounts of cheaper and lower performance storage towards the base of the pyramid. By providing a larger amount of object storage between the PFS and the tape archive, the acquisition and operating budgets should be reduced. The larger amount of object storage will provide more time to transfer data to the tape archive and possibly reduce the need to archive objects (Grider, G 2016, Inman et al. 2014). As jobs are setup to run on the supercomputers, data are moved (possibly) from tape archive to the PFS or the NLDA and just prior to execution, the data can be staged in the BB. During and after execution, both CR datasets and data products from job execution will be stored on the BB and then copied to the PFS. Rather quickly, data may be moved from PFS to NLDA where it will be eventually curated and possibly copied to the tape archive. Two goals in designing the exascale storage system will be to reduce the demand for the top tier BB and PFS components for storage capacity and to reduce the demand for bandwidth for the tape archive storage system. An effective NLDA design will reduce the amount of PFS needed while giving users more time to curate the data, likely reducing the demand for tape archive storage bandwidth and capacity. Eventually, storage resources used on the NLDA will be released so they may be reused by other users. In addition, the lifetime of data on the NLDA will be very short with respect to tape archive storage but long with respect to data stored on PFS. Data protection requirements will not likely extend beyond project work flow needs; lifetime requirements will be months and not years.

The key contribution of this paper is as follows. To perfect the use of cloud storage technologies for HPC, we study the architecture for interfacing cloud storage between the HPC PFS and the archive storage. In this paper we show that computing erasure coding for a high degree of sharding on the Ceph Object File System (Weil et al. 2006) with GPUs outperforms a modern Intel ${ }^{\circledR}$ CPU, opening the potential for new ways to architect such storage system based on Ceph. For use cases where data are moved to object storage systems via single points of mediation, such as the File Transfer Appliances (FTAs) as in the LANL Trinity system, these mediators may be equipped with GPUs to perform erasure encoding and recovery at high speed and utilization. The literature uses the term "stripe" for a set of data that is protected by RAID or an erasure coding implementation. The stripe is divided into $k$ data chunks and protected by $m$ parity or coding chunks. In the literature, "strip" and "shard" are used synonymously and refer to these chunks; in this paper, we will use "shard" to refer to the chunks of a protected stripe of data.

The remainder of this paper is organized as follows. We give an overview of Ceph and erasure coding (see Section 2). In Section 3, we discuss our implementation of the Ceph erasure coding plugin using Gibraltar, a GPU Reed-Solomon coding library. We present our findings from our measurements of our experiments in Section 4. In Section 5 we discuss other research using GPU erasure coding for HPC storage followed by our conclusions in Section 6. 


\section{BACKGROUND}

We utilize Ceph as a platform for our work because of its client libraries and convenient plugin architecture for erasure coding libraries. This structure enabled us to focus our work on the Gibraltar library and to follow the implementation of the Ceph plugin interfaces provided in its erasure code plugin classes. The existing erasure coding plugins in Ceph provide us with well known baselines against which to compare our results.

In previous work, we designed and reduced to practice a library that performs erasure coding on GPU hardware, Gibraltar (Curry et al. 2008, Curry et al. 2011); this approach can further lower the price/performance for storage systems and provide opportunities for performing compute close to the data. One of the consequences of erasure coding in the design of high performance distributed file systems is the high computational and data transfer costs of reconstruction of a failed disk. By including GPUs in the architecture, we provide additional compute resources that can raise the achievable performance. As common disk drive storage capacities have increased from 750 GB in 2006 (Farrance 2006) to 14 TB in 2018 (Western Digital 2018), this architectural performance enhancement will become even more important by off-loading computation for erasure coding to the GPU. Very recently, Seagate announced that it has successfully built a 16 TB Heat Assisted Magnetic Recording (HAMR) hard disk drive (Hruska 2018).

Ceph is a distributed high performance file system that decouples metadata from data and provides a deterministic function for mapping metadata to data location, CRUSH - Controlled Replication Under Scalable Hashing (Weil et al. 2006). It is an object storage system that uses peer-to-peer sharing of a compact hierarchical description of the cluster configuration and replication policy. This innovation distributes the computation to determine replica placement to any member of the cluster, including clients, thus eliminating the serialization that would otherwise result from determining data placement on a centralized metadata service. The CRUSH algorithm uses rule sets to define policies on data placement that result in evenly distributed storage of data across all of the Object Storage Devices (OSDs) in the cluster.

Since the redundant array of inexpensive disks/devices (RAID) was introduced in 1988 by Patterson, Gibson and Katz (Patterson, Gibson, and Katz 1988) that provided an economical way for systems to be more resilient against data loss compared to other options such as pure mirroring, research has continued to provide more techniques for improving availability of data and improving performance. The principle methods for mitigating the loss of data resulting from media or system failure have been replication, RAID and erasure coding. The design choices between these methods must be balanced between 1) the higher cost of storage for replication of $n \times(r+1)$ where $n$ is the size of the data and $r$ is the number of replicas versus 2) the computational cost of parity generation for RAID or erasure coding, or 3) the costs of data transmission. Declustered RAID distributes the shards of data and parity over the subsystem storage disks to balance the work load, especially during repair (Holland and Gibson 1992).

Erasure coding provides a higher degree of durability in that the storage system can survive the loss of a greater number of disks while using less additional storage than replication (Rodrigues and Liskov 2005, Weatherspoon and Kubiatowicz 2002). The property that erasure coding can provide a higher order of redundancy by generating more than two parity disks has been heavily studied by James Plank (Plank, Simmerman, and Schuman 2008, Plank 1997, Plank, Blaum, and Hafner 2013, Plank and Thomason 2004). Another consideration for data reliability is locality. Storage subsystems that replicate data or store parity on direct attached media can provide data storage services incurring a lower communications cost as compared to storage systems that distribute replicas or parity throughout a set of storage nodes that are connected over a high speed network. This is true in the particular case of reconstructing parity for RAID-5, RAID-6 arrays on a storage device where the disks are directly attached as compared to a distributed erasure coding storage system where the minimum set of data or coding shards must be copied over the network and be assembled in a local contiguous memory location for the erasure coding function to recompute the missing data or 
coding. Localized redundancy of data moves the vulnerability to the host systems which is mitigated by a failover mechanism using high availability techniques. Distributing data and parity is less vulnerable to host failure at the loss of locality. Delaying erasure repair on distributed storage systems can reduce the impact of the loss of locality at the risk of reducing the time to data loss. By having larger stripes with proportionately more parity shards we can extend the time to data loss. Erasures may likely be repaired when a user read occurs which will nearly eliminate the communications cost. Gibraltar exploits the massive parallelism that is available in GPUs along with the fast and concurrent memory access provided by the shared and constant memories. All of the computation for the parity is performed as integer multiplication over a finite field by adding the precomputed logarithms stored in the shared and constant memory. For a more in depth description of the Gibraltar algorithm see (Curry et al. 2011). We compared the performance of Gibraltar and ISA-L in (Haddock et al. 2017) where higher parity counts reuse the data sufficiently to amortize the data transfer cost over the PCI bus.

\subsection{System Requirements}

The Campaign Storage System acquired by the Los Alamos National Laboratory (LANL) for the Trinity Super Computer provides requirements for an NLDA. The first phase of Trinity required the NLDA to have about $25 \mathrm{~PB}$ capacity with future expansion capability, bandwidth between 20 to $25 \mathrm{~GB} / \mathrm{s}$, which should increase with capacity, erasure coding for reliability, the use of archive-grade hard disk drives, and performance gains through large scale parallel access. Also, two relaxed constraints, the files stored in the campaign storage system will not be updated in place nor is the system intended for high duty cycle workloads. LANL has also indicated they needed capability to store 1 PB sized checkpoints in the near future (Lamb 2015). With 25 FTAs, these requirements imply that each should move $1 \mathrm{~GB} / \mathrm{s}$ of data.

\section{Ceph ERASURE CODING PLUGIN IMPLEMENTATION FOR GIBRALTAR}

Ceph provides a well-defined interface for integrating erasure coding libraries into the product. This mechanism provides a means to incorporate new erasure coding libraries into the Ceph file system. The plugin architecture is modularized into two functional areas: registration of erasure coding profiles and the interface for the erasure coding/decoding services of the library. Gibraltar can theoretically provide up to 256 data and coding shards in a stripe (Curry et al. 2011). Ceph erasure coded profiles can be constructed with many combinations of $k$ and $m$.

The Ceph ErasureCodePlugin class is subclassed in our work in order to instantiate a Gibraltar instance; this instance is configured according to the parameters provided by the Ceph administrator command to create an erasure code profile. Gibraltar uses the NVIDIA ${ }^{\circledR}$ CUDA $^{\circledR}$ library (NVIDIA 2017) to offload computation and retrieve results from the K40 GPU in our system. The subclass ErasureCodePluginGibraltar calls the Gibraltar gib_cuda_driver function to initialize a CUDA ${ }^{\circledR}$ context for the profile. The profile can then be used to create an erasure coded pool in Ceph.

The Ceph ErasureCode class is subclassed in our work to implement the Ceph ErasureCodeInterface functions for the Gibraltar library. We modified the Gibraltar erasure code library application programmer interface (API) to make it compatible with the Ceph architecture. Ceph uses a bufferlist data structure and aggregates $k+m$ shards for each erasure coded stripe where each shard is referenced by a pointer to the head of the list data structure for the shard. The call to Gibraltar has been modified to provide an array of these pointers and the logic copies each shard of data onto a contiguously allocated GPU memory block. The outputs of the coding or decoding are copied back to the Ceph bufferlist data structures in a similar way. The plugin appends $m$ coding shards onto the Bufferlist object and includes the pointers to these data structures in the array. 


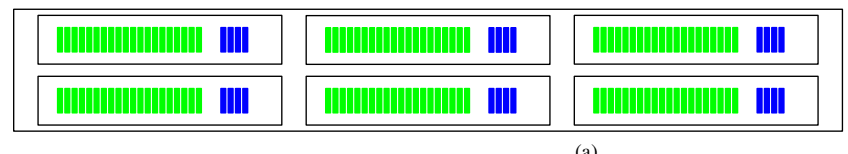

(a)

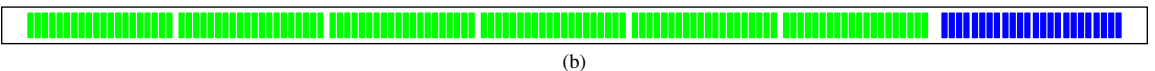

Figure 1: Comparison between two stripe sizes. In a) The current Trinitiy configuration, $\mathrm{k}=20, \mathrm{~m}=4,24$ shards per stripe set, total storage available is $k \times$ shard size $=$ total bytes per stripe. In $\mathrm{b}$ ) our example configuration $k^{\prime}=120, m^{\prime}=24,144$ shards per stripe set, total storage available is $k^{\prime} \times$ shard size $=$ total bytes per stripe. Where $k^{\prime}$ is $6 \times k$ in our example.

\subsection{Architecture}

We have implemented erasure coding on the client nodes of the Ceph cluster using the Ceph C++ Client API. These clients perform the role of the FTAs used by the Hierarchical Storage Management System (HSM). By taking advantage of a higher degree of sharding that can be achieved by Gibraltar and ISA-L for the erasure coded stripes, we can increase the number of parity shards in a stripe without impacting the user experience and without increasing the required storage space. Having a larger number of coding shards will reduce the urgency to repair erasures which can enable us to take a lazy approach to repair. To illustrate the advantage for larger stripes we give an example in Figure 1.

The architecture for exascale and beyond that we propose exploits the single point of mediation provided by the FTAs to perform erasure coding, encryption and other operations on the data as it moves through on its way to a new storage location. We have chosen to implement our solution by using the Ceph client API which provides a granular interface and an opportunity for us to insert our own erasure coding and encryption methods into the dataflow on the FTAs. We exploit the asynchronous write and read methods of the Ceph API to increase parallelism and maximize bandwidth. We have implemented a write pipeline where data flows from a stripe creation thread to to the erasure coding thread. From the erasure coding thread the data moves to the Ceph asynchronous write thread. Each write provides a callback to notify the application when the data are safely stored in the object. The read pipeline is similar where the first thread submits an asynchronous read to the object store. A callback notifies the application when the data are present on the FTA. Data shards are assembled into stripes and moved to the erasure decoding (repair) thread. Once the stripe of data has been repaired, it is ready for use by the user. We have illustrated HPC Storage architecture including our NLDA ideas in our poster (Haddock et al. 2017).

\section{EVALUATION AND MEASUREMENT}

Here, we discuss the configuration of the system used to perform the experiments and then we show the results that we obtained. First we measure write and read performance of the native Ceph system to provide a baseline for comparing the results we obtain using our FTA based methods. Next, we measure the write and read performance using a higher degree of sharding. In the remaining experiments we measure the write and read performance of our encryption feature. We perform our experiments using the Gibraltar library and the Intel ${ }^{\circledR}$ ISA-L.

\subsection{System Description}

We conducted our measurements on Dell R730 servers with an NVIDIA ${ }^{\circledR}$ K40m GPU installed in each FTA. Table 1 lists the common configuration of our cluster. The FTAs and Object Storage Servers (OSS) 
are interconnected via the $10 \mathrm{~Gb} / \mathrm{E}$ network fabric implemented with a Dell Force 10, S4810P switch. Each node has two SFP+ $10 \mathrm{GbE}$ interfaces which are bonded together to provide $20 \mathrm{~Gb} / \mathrm{s}$ total bandwidth. There are four OSS nodes, two FTA nodes configured as shown with a third FTA node having dual Intel ${ }^{\circledR}$ Xeon $^{\circledR}$ E5-2660 v3 @ 2.60GHz. All nodes have Hyperthread-enabled and run 40 threads. We are using forty-eight 6 TB SAS disk drives on each OSS in the test configuration, a total of 192 disks, to provide about 1 PB of total storage in the Ceph file system. The $1 \mathrm{~Gb} / \mathrm{s}$ network is used for management and control.

Table 1: Dell R730 File Transfer Nodes.

\begin{tabular}{ll}
\hline CPUs & 2x Intel ${ }^{\circledR}$ Xeon ${ }^{\circledR}$ E5-2650 v3 @ 2.3 GHZ (Hyperthread-enabled: 40 threads) \\
RAM & 128 GB 2133 MT/s RDIMM \\
Network & Intel ${ }^{\circledR}$ X520 DP 10Gb DA/SFP+, I350 DP 1Gb Ethernet \\
System Drives & 2x 300 GB 10K SAS 2 \\
\hline
\end{tabular}

\subsection{Erasure Code Generation and Reconstruction Performance}

We reported our findings from four experiments in (Haddock et al. 2017) that compared erasure coding and repair performance between the Gibraltar library and the Intel ${ }^{\circledR}$ Storage Acceleration Library (ISA-L) (Tucker 2014). We use threading to create task based SIMD parallelism for the ISA-L. Herein we report results from experiments that we have conducted to measure the performance of using multiple ISA-L encoding threads in parallel for erasure coding and repair. We show that 10 concurrent threads of ISA-L encoding is comparable in performance with the Gibraltar library using 1 concurrent thread. Memory usage on the FTA is higher for the ISA-L implementation because buffers must remain alive until the data are acknowledged as being stored to the Ceph object file system by the Ceph API. For example, using a maximum stripe length of $1 \mathrm{GiB}$ plus an additional $20 \%$ to store erasure coding data, 10 threads of ISA-L required an additional $9 \mathrm{GiB}$ of memory compared to a single Gibraltar thread. In addition, an increase in the latency for the ISA-L benchmark due to the time for the ISA-L implementation to encode or repair the first stripe; Gibraltar is about 6 times faster than a single ISA-L thread with K=120 and M=24 (Haddock et al. 2017).

The results shown were measured by running write tests followed by read tests using 3 FTA nodes and 4 OSS nodes as we have described in Table 1. For all of the tests we cleared the cache on all 7 nodes using a standard procedure for benchmarks on Linux systems. The benchmarks were run using Ceph version 10.2.10, CentOS 7.5, and kernel version 3.10.0-693.11.1.

\subsection{Baseline Performance using Ceph Erasure Coded Pool}

To establish a baseline, we measured the read and write performance of our cluster using a native Ceph erasure coded pool. The pool was created with $\mathrm{K}=20, \mathrm{M}=4$, and the ISA-L Cauchy algorithm. This was the only pool on the cluster and we created 6,144 page groups over 190 OSDs as required by Ceph. We wrote and read data using the 3 FTA nodes. In this experiment, we wrote 540 objects of size 83886080 bytes concurrently from each FTA for the first test and then read them back in the second test writing and reading a total of $135.9 \mathrm{GiB}$ in each test. These tests showed an average aggregate write bandwidth of $217.9 \mathrm{MiB} / \mathrm{s}$ and an average aggregate read bandwidth of $222.5 \mathrm{MiB} / \mathrm{s}$.

We also measured the performance of encoding with Gibraltar and ISA-L on the FTA nodes where we encoded 4,200 stripes of size 83886080 bytes in a shorter amount of time, writing and reading $2.5 \mathrm{TiB}$ on each test with an average aggregate write and read bandwidth above $2 \mathrm{GiB} / \mathrm{s}$ for the cluster. These network write and read bandwidth performance results are shown in Figure 2 where we compare the results of these tests with the baseline measured in the previous experiment. It is important to note that with the native Ceph 
Haddock, Curry, Bangalore and Skjellum
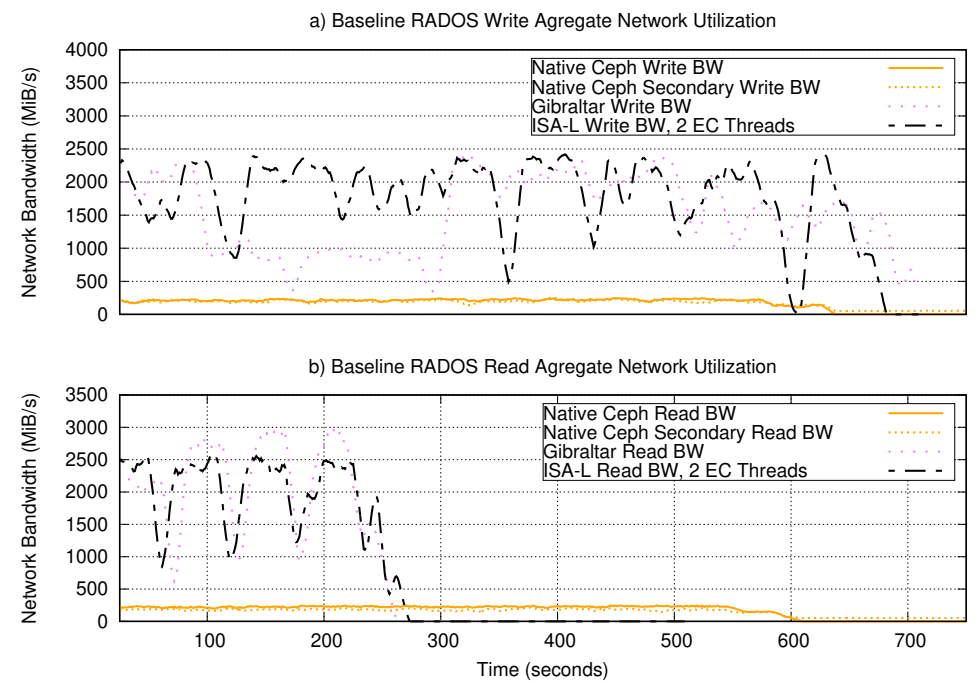

Figure 2: Comparison of Ceph network write (graph a) and read (graph b) performance between native Ceph erasure Coded pool with erasure coding on the FTAs. $\mathrm{K}=20, \mathrm{M}=4$, one thread of Gibraltar encoding, two threads of ISA-L encoding.

erasure coding implementation that data is transferred over the network two times (with the exception of the object(s) stored on the encoding OSS) which nearly doubles the network bandwidth cost. This occurs during reading and writing. The data transfer between the FTA and the OSS is shown as the "Primary" line while the additional writes and reads are shown on the chart as "Secondary" line.

The CPU utilization for writing and reading was measured and is shown in Figure 3. Note that the read performance was done without any repair operations in progress. The CPU utilization for the writing benchmark shows peaks as high as 16\% with an average around 10\% while the average reading CPU utilization is about $8 \%$. The CPU utilization on the client nodes for reading and writing using the Ceph erasure coded pool is very light.

\subsection{Comparison between native Ceph Erasure Coding and Erasure Coding on the File Transfer Appliances}

In Table 2 we compare the performance between three configurations. Our baseline was the native Ceph erasure coding pool using the Cauchy algorithm of the ISA-L. The second is the FTA erasure coding implementation using the ISA-L and the third is the Gibraltar Erasure Coding Library implemented on the FTA. We compare the performance between write bandwidth, read bandwidth and CPU utilization.

\subsection{Performance of Erasure Coding on File Transfer Nodes}

Our implementation of erasure coding for nearline object storage for HPC on the FTAs was tested with several benchmarks which measured write bandwidth, read bandwidth and CPU utilization. We compared the performance using our Gibraltar library and the ISA-L library by transferring $604 \mathrm{GiB}$ of data on each FTA. First the data are written and then they are read back. The total data moved in each test is about 1.8 TB. In Figure 4 we compare the write and read performance between one thread with the Gibraltar library implementation and 5 threads with the ISA-L implementation. 
Haddock, Curry, Bangalore and Skjellum
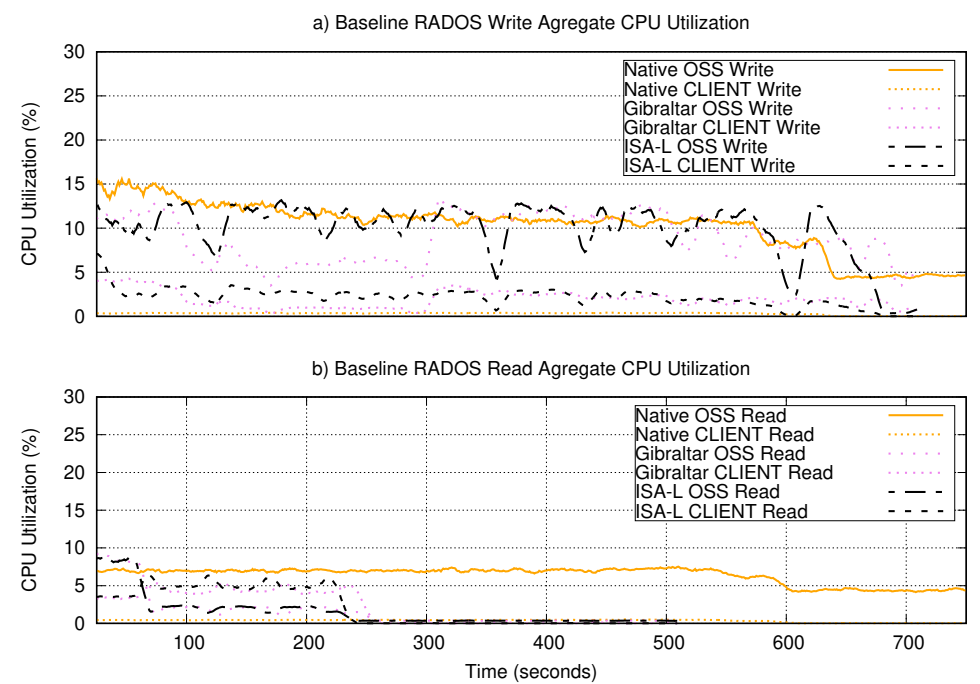

Figure 3: Comparison of Ceph CPU write (graph a) and read (graph b) performance between native Ceph erasure coded pool with erasure coding on the FTAs, $\mathrm{K}=20, \mathrm{M}=4$, one thread of Gibraltar encoding, two threads of ISA-L encoding.

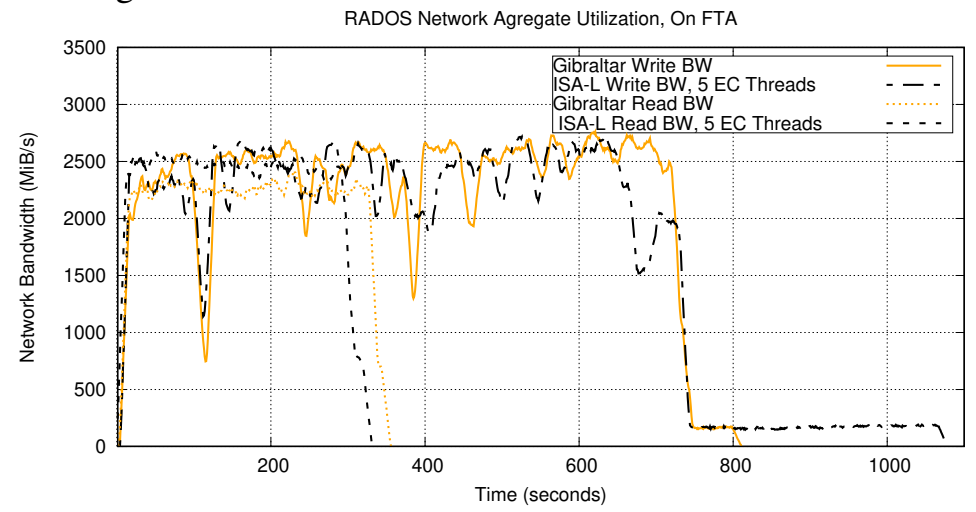

Figure 4: RADOS network performance with write and read of 600 stripes, $K=120, M=24$, shard size 8388608 bytes, one thread of Gibraltar encoding compared with five threads of ISA-L encoding.

Read network bandwidth performance between the two are nearly equivalent. In the read benchmark the task was to read $k$ data shards and reconstruct $m$ parity shards to simulate the worst case scenario. The results show that both implementations are capable of providing the data to the user with little latency other than the startup of the pipeline. Once the first stripes are repaired and sent to the user, subsequent stripes continue to flow at nearly $800 \mathrm{MB} / \mathrm{s}$ per FTA node. The CPU graph Figure 5 shows that the repair for ISA-L continues nearly 200 seconds after the data transfer has finished. Increasing the number of erasure coding threads from 5 to 10 results in the repair of 24 erasures at the same rate as reading from the object store in this experiment.

The CPU utilization of the write and read benchmarks for the FTA erasure coding using the ISA-L and Gibraltar library is shown in Figure 5. Because the erasure coding work is off loaded to the Nvidia K40 GPU, the FTA host CPU utilization remains low. The FTA utilization goes to $1.7 \%$ during the write and read erasure coding. For ISA-L, writing with 5 execution threads, the FTA host CPU utilization averages $2.5 \%$. For reading in this configuration, the FTA host CPU utilization averages $1.7 \%$. Note that with both erasure coding methods, the OSS CPU utilization does not change with writes running about $11 \%$ utilization and reads running about $1 \%$ utilization. 
Haddock, Curry, Bangalore and Skjellum
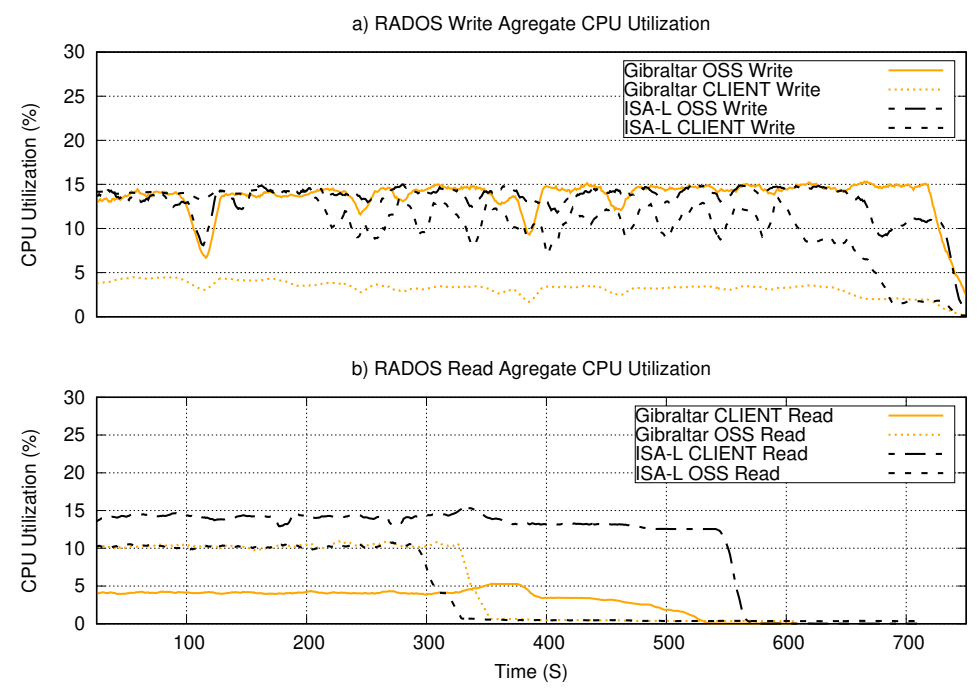

Figure 5: RADOS CPU performance with write and read of 600 stripes, $K=120, M=24$, shard size 8388608 bytes, one thread of Gibraltar encoding compared with five threads of ISA-L encoding. Write performance shown in graph a and read performance shown in graph $b$.

Table 2: Comparison of Erasure Coding Performance.

\begin{tabular}{llll}
\hline Property & $\begin{array}{l}\text { Ceph } \\
\text { Pool }\end{array}$ & $\begin{array}{l}\text { FTA } \\
\text { ISA-L }\end{array}$ & $\begin{array}{l}\text { FTA } \\
\text { Gibraltar }\end{array}$ \\
\hline Bandwidth Write $^{\mathrm{a}}$ & .22 & 1.9 & 1.7 \\
Bandwidth Read $^{\mathrm{a}}$ & .23 & .79 & .84 \\
FTA Write CPU & $1 \%$ & $2.5 \%$ & $1.7 \%$ \\
FTA Read CPU & $1 \%$ & $1.7 \%$ & $1.7 \%$ \\
OSS Write CPU & $11 \%$ & $10.9 \%$ & $8.8 \%$ \\
OSS Read CPU & $7 \%$ & $.9 \%$ & $.9 \%$ \\
\hline
\end{tabular}

${ }^{\mathrm{a}}$ Bandwidth is in $\mathrm{GB} / \mathrm{S}$.

\section{PREVIOUS WORK}

Ceph provides an erasure coding plugin class as an extensible way for erasure coding libraries to be implemented in the product. Currently, in release 12.0.0, there are four erasure coding libraries implemented, namely: Jerasure (Plank, Simmerman, and Schuman 2008), ISA-L (Tucker 2014), 1rc (Papailiopoulos and Dimakis 2014) and Shingled Erasure Code (SHEC) (Miyamae, Nakao, and Shiozawa 2014). Our study has shown that the Gibraltar library (Curry et al. 2010) can perform about half as well as these libraries for a sharding degree less than 40 while Gibraltar performs better than these for greater degrees of sharding. Khasymski et al., showed that GPU-assisted erasure coding and reconstruction can be performed on the Lustre file system. Their work was implemented with a software shim to integrate into Lustre. Their work was limited to implementing a RAID-6, providing $m=2$, demonstrated the feasibility and showed that providing erasure coding can provide strong fault tolerance (Khasymski et al. 2012) without having to depend on RAID subsystems and failover mechanisms for availability. ZFS (Stearman 2015), a file system developed by Sun Microsystems and has been used to provide a reliable storage layer under Lustre using locally attached block devices. Stearman found that the performance was lower on ZFS managed disks as compared to using SAN storage systems. 


\section{CONCLUSION}

We have implemented the Gibraltar GPU erasure coding library (Curry et al. 2011) as a plugin in the Ceph product and shown that it provides high bandwidth for large degrees of sharding. We have implemented erasure coding as a Ceph client and demonstrated the performance capability of moving data at near 700 $\mathrm{MB} / \mathrm{s}$ between an FTA and a Ceph storage cluster. We have shown that multiple threads can be used to erasure code data with CPU algorithms to achieve the required bandwidth. We have demonstrated a high throughput during reading of data from the Ceph storage cluster with 24 erasures.

\section{FUTURE WORK}

Performing erasure coding on FTAs provides other opportunities for data reuse. We are currently working on implementing encryption on the data as it flows from source to storage. Encryption is done on each shard concurrently providing large amounts of parallelism. Other candidates for services on the FTAs are data compression and feature detection. The design of our framework will lend itself to integration with products like PFTool and MarFS (Chen, Grider, and Montoya 2017). Analysis of the costs of acquiring and operating NLDA storage systems will be helpful in minimizing expense of using Exascale systems. The development of a model of disk drive failure will enable NLDA systems to take full advantage of lazy repair policies to reduce costs. Benchmarking should be performed on a system where there is a higher ration of OSSs to FTAs which should be more consistent with a production design.

\section{ACKNOWLEDGMENTS}

This material is based upon work supported by the National Science Foundation under Grants Nos. ACI1541310, CNS-0821497, CNS-1229282, CCF-1562306, OAC-1642078, and CCF-1822191. Any opinions, findings, and conclusions or recommendations expressed in this material are those of the authors and do not necessarily reflect the views of the National Science Foundation.

This material is based upon work supported by Sandia National Laboratories. Sandia National Laboratories is a multimission laboratory managed and operated by National Technology and Engineering Solutions of Sandia, LLC., a wholly owned subsidiary of Honeywell International, Inc., for the U.S. Department of Energy's National Nuclear Security Administration under contract DE-NA-0003525.

\section{REFERENCES}

Bent, J., B. Settlemeyer, and G. Grider. 2016. "Serving Data to the Lunatic Fringe”. ;login: vol. 41 (2), pp. 34-38.

Chen, H., G. Grider, and D. R. Montoya. 2017, April. "An Early Functional and Performance Experiment of the MarFS Hybrid Storage EcoSystem”. In 2017 IEEE International Conference on Cloud Engineering (IC2E), pp. 59-66. IEEE.

Curry, M. L., A. Skjellum, H. Lee Ward, and R. Brightwell. 2008, April. "Accelerating Reed-Solomon coding in RAID systems with GPUs". In 2008 IEEE International Symposium on Parallel and Distributed Processing, pp. 1-6. IEEE.

Curry, M. L., A. Skjellum, H. L. Ward, and R. Brightwell. 2011, December. "Gibraltar: A Reed-Solomon coding library for storage applications on programmable graphics processors". Concurrency and Computation: Practice and Experience vol. 23 (18), pp. 2477-2495.

Curry, M. L., H. L. Ward, A. Skjellum, and R. Brightwell. 2010, September. "A Lightweight, GPU-Based Software RAID System". In 2010 39th International Conference on Parallel Processing, pp. 565-572. San Diego, CA, USA, IEEE. 
Farrance, Rex 2006, September. "Timeline: 50 Years of Hard Drives".

Grider, G 2016, April. "HPC Storage and IO Trends and Workflows".

Haddock, W., M. L. Curry, P. V. Bangalore, and A. Skjellum. 2017, June. "GPU Erasure Coding for Campaign Storage". In International Conference on High Performance Computing, pp. 145-159. Frankfurt, Germany, ISC, Springer.

Holland, M., and G. A. Gibson. 1992. "Parity declustering for continuous operation in redundant disk arrays". In ASPLOS-V Proceedings - Fifth International Conference on Architectural Support for Programming Languages and Operating Systems, Boston, Massachusetts, USA, October 12-15, 1992, edited by B. Flahive and R. L. Wexelblat, Volume 27. ACM, ACM Press.

Hruska, J. 2018, December. "Seagate Announces It's Successfully Built 16TB HAMR Drives".

Inman, J., G. Grider, and H. B. Chen. 2014, June. "Cost of Tape versus Disk for Archival Storage". In 2014 IEEE 7th International Conference on Cloud Computing, pp. 208-215. Anchorage, AK, USA, IEEE.

Khasymski, A., M. M. Rafique, A. R. Butt, S. S. Vazhkudai, and D. S. Nikolopoulos. 2012, August. "On the Use of GPUs in Realizing Cost-Effective Distributed RAID". In 2012 IEEE 20th International Symposium on Modeling, Analysis and Simulation of Computer and Telecommunication Systems, pp. 469-478. Washington, DC, USA, IEEE.

Lamb, K. 2015, August. "Trinity Campaign Storage and Usage Model”.

McCorkle, M. 2018, June. “ORNL Launches Summit Supercomputer”. https://www.ornl.gov/news/ornllaunches-summit-supercomputer.

Miyamae, T., T. Nakao, and K. Shiozawa. 2014, October. "Erasure Code with Shingled Local Parity Groups for Efficient Recovery from Multiple Disk Failures". In 10th Workshop on Hot Topics in System Dependability (HotDep 14). Broomfield, CO, USENIX Association.

Morgan, T. P. 2018, March. "The Roadmap Ahead For Exascale HPC In The US". https://www.nextplatform.com/2018/03/06/roadmap-ahead-exascale-hpc-us/.

Morton, D. 2017, January. "Trinity: DataManagement Scheme and Performance". In 55th AIAA Aerospace Sciences Meeting. AIAA SciTech Forum, American Institute of Aeronautics and Astronautics.

NVIDIA 2017, March. "CUDA Parallel Computing Platform".

Papailiopoulos, D. S., and A. G. Dimakis. 2014. "Locally repairable codes". IEEE Transactions on Information Theory vol. 60 (10), pp. 5843-5855.

Patterson, D. A., G. Gibson, and R. H. Katz. 1988. "A Case for Redundant Arrays of Inexpensive Disks (RAID)". In Proceedings of the 1988 ACM SIGMOD International Conference on Management of Data, SIGMOD '88, pp. 109-116. New York, NY, USA, ACM.

Plank, J. L., S. Simmerman, and C. D. Schuman. 2008. "Jerasure: A Library in C/C++ Facilitating Erasure Coding for Storage Applications". Technical Report Technical Report CS-08-627, University of Tennessee, Knoxville, TN 37996.

Plank, J. S. 1997, September. "A Tutorial on Reed-Solomon Coding for Fault-Tolerance in RAID-like Systems". Software - Practice \& Experience vol. 27 (9), pp. 995-1012.

Plank, J. S., M. Blaum, and J. L. Hafner. 2013, February. "SD codes: erasure codes designed for how storage systems really fail.". In 11th USENIX Conference on File and Storage Technologies (FAST 13), pp. 95-104. San Jose, CA, USA, USENIX Association.

Plank, J. S., and M. G. Thomason. 2004. "A practical analysis of low-density parity-check erasure codes for wide-area storage applications". In Dependable Systems and Networks, 2004 International Conference on, pp. 115-124. IEEE. 
Rodrigues, R., and B. Liskov. 2005. "High availability in DHTs: Erasure coding vs. replication". In Peerto-Peer Systems IV, pp. 226-239. Springer.

Stearman, D. 2015. "ZFS on RBODs-Leveraging RAID Controllers for Metrics and Enclosure Management". Technical report, Lawrence Livermore National Lab.(LLNL), Livermore, CA (United States).

Tucker, Greg 2014, April. "ISA-L open source v2.14 API doc".

Weatherspoon, H., and J. D. Kubiatowicz. 2002. "Erasure coding vs. replication: A quantitative comparison”. In Peer-to-Peer Systems, pp. 328-337. Springer. 10.1007/3-540-45748-8_31.

Weil, S. A., S. A. Brandt, E. L. Miller, D. D. E. Long, and C. Maltzahn. 2006. "Ceph: A scalable, highperformance distributed file system". In Proceedings of the 7th symposium on Operating systems design and implementation, pp. 307-320. USENIX Association.

Weil, S. A., S. A. Brandt, E. L. Miller, and C. Maltzahn. 2006, November. "CRUSH: Controlled, Scalable, Decentralized Placement of Replicated Data". In SC 2006 Conference, Proceedings of the ACM/IEEE, pp. 31-31. Tampa, FL, USA, IEEE.

Western Digital 2018, September. "Ultrastar ® DC HC530”.

\section{AUTHOR BIOGRAPHIES}

WALKER HADDOCK is a Ph.D. student at the University of Alabama at Birmingham. His research interests are HPC systems architecture. His email address is whaddock@uab.edu.

MATTHEW L. CURRY received his Ph.D. from the University of Alabama at Birmingham. He is a research and development staff member at Sandia National Laboratories. His research interests include parallel and distributed storage systems. His email address is mlcurry@ sandia.gov.

PURUSHOTHAM V. BANGALORE is a Professor in the Department of Computer Science, University of Alabama at Birmingham. He received his Ph.D. from MSU. His email address is puri@uab.edu.

ANTHONY SKJELLUM is a Professor of Computer Science, Chair of Excellence, and Director, SimCenter at the University of Tennessee at Chattanooga, where he continues work in HPC and Cybersecurity, with strong emphases on IoT and blockchain technologies. He received received his Ph.D. from Caltech. His email address is tony-skjellum@utc.edu. 